Revue européenne des sciences sociales

European Journal of Social Sciences

54-1 | 2016

Les usages sociaux des sciences du vivant

\title{
Race, racisme, racialisation : que nous disent les discours?
}

Race, racism, racialization: what can we learn from discourse?

\section{Marie Peretti-Ndiaye}

\section{(2) OpenEdition}

12 Journals

Édition électronique

URL : http://journals.openedition.org/ress/3459

DOI : $10.4000 /$ ress.3459

ISBN : $1663-4446$

ISSN : $1663-4446$

Éditeur

Librairie Droz

Édition imprimée

Date de publication : 15 mai 2016

Pagination : 103-128

ISSN : 0048-8046

Référence électronique

Marie Peretti-Ndiaye, "Race, racisme, racialisation : que nous disent les discours ? 》, Revue européenne des sciences sociales [En ligne], 54-1 | 2016, mis en ligne le 15 mai 2019, consulté le 21 décembre 2020. URL : http://journals.openedition.org/ress/3459 ; DOI : https://doi.org/10.4000/ress. 3459

(c) Librairie Droz 


\title{
RACE, RACISME, RACIALISATION: QUE NOUS DISENT LES DISCOURS?
}

\author{
MARIE PERETTI-NDIAYE \\ CADIS - CNRS-EHESS, Paris \\ marie.peretti.ndiaye@gmail.com
}

Résumé. L'enjeu de cet article est d'examiner les liens entre race, racialisation et racisme à la lumière de matériaux empiriques afin d'envisager les formes contemporaines du rejet en France. II s'agit ainsi d'appréhender une réalité multiforme dont l'expression est encadrée par un ensemble de règles et de normes sociales. Fondé sur une analyse de discours recueillis dans les années 2000 dans différentes régions françaises, cet article s'appuie sur la distinction, opérée par Michel Wieviorka, entre racisme classique, racisme d'infériorisation et racisme différencialiste. Après avoir envisagé la manière dont ces logiques peuvent se déployer dans les discours racistes, il questionne les spécificités du racisme en interrogeant ses liens avec l'idée de race puis en soulignant ses différences avec les formes renouvelées de racialisation observées en France.

Mots-clés: analyse de discours, race, racialisation, racisme, racisme différentialiste.

\begin{abstract}
This article aims at examining the links between race, racialization and racism in the light of empirical data in order to analyze the contemporary forms of rejection in France. It considers a multifaceted reality, whose expressions are framed by rules and societal norms. Based on discourse analysis, it draws on Michel Wieviorka's distinction between classical racism, inferiorization and differentialism. After showing that these logics can coexist, it looks at racism specificities by questioning their links with the idea of race. Then, it highlights the differences between neo-racism and new racialization.
\end{abstract}

Keywords: differentialist racism, discourse analysis, race, racism, racialization. 
Des violences islamophobes répertoriées par le Collectif contre l'Islamophobie en France (Dorémus et Mohammed, 20I3) à la caricature de Christiane Taubira parue en Une du journal Minute le i3 novembre 20I3, des actes de rejet ont eu, ces dernières années, une visibilité accrue dans l'espace public. Leur banalisation et les formes de légitimation qui en découlent suscitent, çà et là, des inquiétudes. Le spectre du «racisme» a notamment été brandi pour caractériser ces événements. Dans un communiqué de presse faisant suite à la parution du rapport 20ı2, la Commission nationale consultative des droits de l'homme s'inquiète ainsi du fait que «la société française semble plus perméable aux phénomènes d'intolérance et de racisme» (CNCDH, 20I3).

Un tel usage du mot racisme peut toutefois être questionné : le concept est-il opératoire pour analyser les faits ainsi embrassés ? Doit-on considérer, a contrario, qu'il est trop englué dans des enjeux politiques ou médiatiques pour permettre d’appréhender sereinement de tels phénomènes? Dans quelle mesure ce vieux terme des sciences sociales peut-il encore constituer une ressource pour les chercheurs? Ne les condamne-t-il pas à mener des réflexions par trop éculées, peu en phase avec les mutations induites par les évolutions scientifiques ou l'ethnicisation de la société (Bertheleu, 2007)?

Employé principalement à partir des années 1930 pour désigner une idéologie dont les locuteurs se réclament - celle de la croyance en des différences génétiques et des pratiques d'évitement qui lui sont associées -, le «racisme» désigne aujourd'hui des actes et des perceptions fondées sur la caractérisation d'«un ensemble humain par des attributs naturels, eux-mêmes associés à des caractéristiques intellectuelles et morales qui valent pour chaque individu relevant de cet ensemble» (Wieviorka, 1998, p. 7). Ce processus de biologisation du social et de réification des différences (Guillemin, 2002) semble, en outre, perdurer indépendamment de la croyance en l'existence des «races». À ce titre, le concept de «racisme» est toujours mobilisé dans le champ académique. Une recherche effectuée en janvier 20I4 dans la bibliothèque numérique de la Bibliothèque Nationale de France Gallica ${ }^{1}$ indique 2 I77 occurrences 
comportant le terme «racisme», dont I 237 renvoient à des sources parues depuis 200I. La même recherche réalisée dans la base de données de Cairn ${ }^{2}$ révèle, quant à elle, l'existence de 7 59I occurrences depuis 200I. L'usage du terme n'en fait pas moins débat dans le champ scientifique (Noiriel, 2007b) et l'on retrouve, au cœur des controverses, un scepticisme quant à l'usage d'un concept originellement conçu en référence à des différences génétiques, physiques et morales dont on sait qu'elles n'ont d'autre existence que fantasmée.

Peut-on encore parler de racisme aujourd'hui alors que la croyance en l'existence de races s'est largement estompée au cours du $\mathrm{xx}^{\mathrm{e}}$ siècle, que les progrès de la génétique ont jeté le discrédit sur les théories racistes (Poli, 2005) ? Ce concept est-il toujours approprié face à la banalisation de l'usage des catégories ethnico-raciales dans les espaces public et privé et jusqu'au cœur des institutions (Sajas, 20ı) ? Ne doit-on pas plutôt parler d'une racialisation exempte de racisme?

L'enjeu de cet article est de revenir sur les liens entre race, racialisation et racisme à la lumière de matériaux empiriques afin d'envisager les formes contemporaines du rejet en France. Après avoir situé ces concepts dans le champ des débats théoriques, nous chercherons à saisir les traits d'une réalité multiforme dont l'expression est encadrée par un ensemble de normes et traversée par des courants transgressifs - autrement dit à appréhender des manifestations fuyantes et plurielles. L'analyse de plusieurs extraits de discours racistes recueillis auprès de différents acteurs de la vie sociale dans le cadre d'une recherche doctorale menée en Corse au cours des années 2000 et d'études socio-urbaines réalisées à Lille, Paris et sa région, Toulouse et Reims constituera le fil d'Ariane de cette réflexion?3. Elle nous conduira, par la suite, à distinguer

2 Voir <http://www.cairn.info/>.

3 La recherche doctorale réalisée en Corse portait spécifiquement sur le racisme; elle a donné lieu à la réalisation de 96 entretiens semi-directifs et non directifs auprès de différents acteurs de la vie sociale et politique. Les enquêtes réalisées à Lille, Reims, Toulouse et la région parisienne ont, quant à elles, été réalisées dans le cadre de recherches portées par l'École des hautes études en sciences sociales («enquête PRESAL» pour Perception des risques en santé environnement sur les agglomérations lyonnaise et lilloise, 20II-20I2) et le laboratoire de sociologie générative du Centre scientifique et technique du bâtiment - CSTB («Étude de 
puis à ré-agréger les trois logiques racistes identifiées par Michel Wieviorka : le racisme classique, le racisme d'infériorisation et le racisme différentialiste. Cela nous amènera, pour finir, à mettre en évidence les spécificités françaises du racisme en questionnant ses liens avec l'idée de race puis en soulignant ses différences avec les formes renouvelées de racialisation observées en France.

\section{I.DE L'ALTÉRISATION AU RACISME}

Discours publics et privés (Noiriel, 2007a) témoignent de la prégnance des processus d’altérisation dans l'espace public français. Ils font, en cela, écho à la montée en puissance du thème de la différence observée sur différents terrains d'enquête (Wieviorka, I992; Felouzis, 2003; Sajas, 2010) : à l'école comme au travail, plusieurs individus font l'expérience de «l'imputation d'une extranéité » (Poiret, 20I I, p. i16). Lorsque cette expérience se charge de l’assignation de caractéristiques raciales, elle relève alors de la racialisation et peut sous-tendre un ordre social raciste. Quels liens entretiennent ces différents processus? Peut-on postuler l'existence d'un continuum qui conduirait de l'altérisation au racisme en passant par l'ethnicisation et la racialisation? Ou, à l'inverse, penser leur disjonction?

recomposition urbaine du groupe d'habitations collectives "Chalet-Solferino" à Reims», 20 I3; «Insécurité et violence dans le quartier du Mirail (Toulouse)», 20I0; «Dynamiques résidentielles et rapport à l'habitat des ménages périurbains», CSTB, 2009; «Étude socio-urbaine. Clichy sous Bois. Quartier du Bas-Clichy», 2009; «Étude socio-urbaine en vue de la requalification de l'environnement du secteur Beaubourg-St Merri à Paris 4e arrondissement», 2008; «Coopération et famille. Mixité des statuts d'occupation, modes d'habiter des résidents et fonctionnement des résidences », 2007-2008). Durant ces enquêtes, qui portaient sur la question du cadre de vie et de l'environnement, 146 entretiens semi-directifs relativement longs (entre une heure trente et trois heures) ont été réalisés et plusieurs matériaux concernant les relations interethniques et le racisme ont été recueillis incidemment, alors que les habitants étaient interrogés, à domicile, sur la question de leurs relations de voisinage ou sur leurs expériences du quartier. Ce sont ces discours spontanés qui sortent quelque peu du cadre premier de ces enquêtes qui seront ici exploités. Les observations réalisées dans le cadre de ma participation, en 2008, au volet de la recherche sur les populations musulmanes dans l'Union européenne concernant le $18^{e}$ arrondissement parisien, réalisée par l'Open Society Institute, ont également nourri ma réflexion. 
Néologisme inspiré de l'anglais othering, le concept d'«altérisation» s'inscrit dans le cadre d'une réflexion sur la dialectique de l'identification (Jenkins, 2000). Il caractérise l'ensemble des processus de construction de l'Autre et embrasse les phénomènes liés à l’adresse, la nomination et, plus largement, la catégorisation des groupes. Les travaux d’auteures féministes ont mis à jour le rôle déterminant du mécanisme de l’adresse dans la constitution des catégories qui constituent l'ordre patriarcal (Butler, 2006, 2004; Austin, 1991 ; Delphy, 2008) : ce qui fonde le pouvoir des Uns est, en premier lieu, la capacité de nommer les Autres (Delphy, 2008). La hiérarchisation est immanente à la division. Ce constat s'applique au champ des relations interethniques. Aux catégories «Noirs», «Blancs» ou «Arabes » parfois mobilisées de manière ludique par les collégiens des quartiers populaires (Sajas, 20ı) sont ainsi étroitement associées des valences, positives ou négatives, et une hiérarchie ${ }^{5}$. C'est d’ailleurs là que réside, pour eux, l'intérêt de ces jeux : dans la possibilité de réduire, en la sur-jouant, la honte attachée à certains de ces termes (Mansouri, 20I3), voire de les resignifier (Butler, 2004). Si ces processus revêtent une importance considérable dans la constitution des États-nations (Räthzel, 1998), le travail de définition des frontières du Même et de l'Autre qui les constitue s'observe, aujourd'hui, dans le cadre plus quotidien des échanges interindividuels. L'ethnicité constitue un principe de division dont l'importance varie d'une société à l'autre (Poutignat et Streiff-Ferrart, 1995). Le concept d'«ethnicisation» caractérise, quant à lui, la montée en puissance de

4 Comme le souligne Christian Poiret, « les termes "race", "racial", "ethnie", "ethnique", de même que les termes utilisés pour désigner l'une ou l'autre de leurs spécifications ("Noirs", "Arabes", "africanité", etc.) » (20II, p. 108) ne constituent en aucun cas des catégories d'analyse ; il s'agit plutôt de catégories descriptives, empruntées aux acteurs de la vie sociale et utilisées pour décrire les relations qu'ils peuvent entretenir.

5 Les deux situations suivantes, observées dans un collège de Montreuil, sont éloquentes à ce propos. La première se déroule à l'heure de la cantine. Une jeune fille scolarisée en troisième, Amélia, est avec quatre de ses amies dans la cours de récréation. Elle prend l'une d'entre elles dans ses bras et affirme en riant et en narguant les autres: «Nous, on est VIP» puis développe, toujours rieuse : «Very Importante Portugaise. Ça marche pas avec les sales Arabes, ça». L'amie visée par l'insulte, rit. La seconde se déroule durant un intercours. Cette fois ce sont des élèves de quatrième qui parlent de leur professeur, M. Ben Mohammed. Ils s'interrogent sur son origine. L'un des élèves raconte: «On lui a demandé de quel pays il était. II a dit que sa mère est bretonne». «Et son père?», questionne un autre élève. «II n'a pas voulu répondre». Les collégiens concluent: «ll doit avoir honte de ses origines». 
ce «processus par lequel la référence ethnique occupe une place prépondérante ou centrale, par préférence [...] ou par rejet» (De Rudder, I99I, p. I43). Parfois employé de façon à occulter les jeux relationnels qui constituent «l'ethnie » comme marqueur différentiel, il renoue alors avec un vieux démon de l’anthropologie : le déterminisme biologique qui absolutise la différence (Guillaumin, 2002 [1972]). Employé comme avatar d'une référence à la «race » désormais taboue, l'«ethnie » relève d'une même démarche d'explication des inégalités. La prise en compte des processus d’assignation et d’autodéfinition peut permettre d'échapper à ce biais déterministe et biologisant (De Rudder, I99I, p. I4I).

Ce déterminisme est caractéristique de la racialisation, entendue comme l'attribution extensive de caractéristiques raciales à des individus, des groupes ou des relations sociales (Omi et Winant, 1986), qui consiste, in fine, à définir un ordre social. Elle peut, à ce titre, être considérée comme le soubassement idéologique du racisme (Poiret, 20II, p. II3). Mais si historiquement, la racialisation découle du besoin de justifier l'esclavage dans un contexte où «affranchissements» et «métissages» menacent d’implosion l'ordre esclavagiste (Bonniol, 2008, p. 23), le terme est aujourd'hui employé pour caractériser la diffusion des catégories ethnico-raciales dans l'espace des interactions, parfois indépendamment de la domination inhérente aux rapports sociaux racistes. La montée en puissance des «cosmétiques ethniques» et autres «produits de niche» mais aussi, plus largement, des catégories ethnico-raciales tend, en effet, à ré-ancrer dans l'espace des relations sociales la référence aux différences phénotypiques. Elle déborde très largement les tabous et les normes intégrées en France après la Seconde Guerre mondiale sous l'égide d'instances internationales telles que l'ONU et l'UNESCO (Balibar, 2005). «Noir», «Métis», «Arabe» ou «Blanc» constituent les occurrences les plus fréquentes de cette catégorisation qui, déjouant les controverses auxquelles ont donné lieu les revendications en termes de «statistiques ethniques» ou de «mesure de la diversité » (Masclet, 20I2), pénètrent jusqu’au cœur des manuels scolaires ${ }^{6}$. La racil'ouvrage de cours élémentaire moyen 1000 problèmes édité par Hachette Éducation (Lucas, Rosa et Leclec'h, 2012, p.77). 
sation constitue le degré extrême de cette assignation raciale (Eberhard, 20ı). À ce stade, les individus racisés n'existent alors plus que «comme l’incarnation de leur groupe d'appartenance» (Poiret, 20I I, p. I07). Dès lors, le concept de racisme s'avère-t-il opératoire pour saisir ces différents aspects? Quels sont, parmi les différents faits abordés, ceux qu'il permet de penser?

Le racisme est un rapport social qui a longtemps pris appui sur la croyance socialement construite en des «races» incarnant les contradictions de l'espace social: aux «Blancs», la «positivité» et la «beauté féminine diaphane et pure» (Laurent et Leclère, 20ızb, p. II), aux «Noirs», l'animalité et les expressions de joie naïves (Bancel et Blanchard, 1998), entre eux, la palette de la «dégénération due à l'éloignement de la zone climatique tempérée » (Blanchard et Boëtsch, 20I3, p. I30). Si la classification de Blumenbach semble, à ce propos, prodromique (Degioanni et Gourjon, 20I3), c'est la «dichotomie Blanc-maître/indigène-opprimé » (Laurent et Leclère, 2oızb, p. ı6) qui constitue le paroxysme d'une telle pensée: la «race» n'y existe que dans un rapport d'opposition ou de différenciation. La généalogie du terme permet d'envisager plus précisément cet aspect. L'étymologie proposée par Léo Spitzer - le terme italien razzio (puis razzia) proviendrait du latin ratio qui signifie «système » ou «procédé» (1948) - est retenue par Maurice Olender (2009). Bien qu’incertaine, elle lui paraît pertinente pour illustrer cette fonction de classement qui ponctue l'histoire de la notion de «race». L'historien met ainsi l'accent sur la fonction rhétorique d'un terme qui dans son sens prémoderne comme dans son acception moderne sert à classifier le genre humain. En France, le terme désigne jusquau XVIII ${ }^{\mathrm{e}}$ siècle la lignée ou filiation, soit cette appartenance familiale qui organise les microsociétés dans l'État. «Vieux mot de la noblesse» (Olender, 2009, p. 15), il induit alors l'idée d’une différenciation sociale liée à la naissance. Le vocable se charge de valences spécifiques dès la fin du XVIII ${ }^{\mathrm{e}}$ siècle : il associe attributs biologiques et culturels, et fait l'objet de théorisations scientifiques (Wieviorka, 1998). Ces évolutions découlent de deux éléments qui marquent l'histoire de la pensée : l'emprise décroissante du religieux au cours des XVIII ${ }^{\mathrm{e}}$ et XIX ${ }^{\mathrm{e}}$ siècles et la recherche, afférente, d'explications du fonctionnement social - et plus spécifiquement des inégalités - centrées 
sur l'homme (Guillaumin, 2002 [1972]). Le racisme permet, dans les discours scientifiques, de rationnaliser ce «nouvel ordre anthropologique» en partie sous-tendu par des grilles de lecture évolutionnistes (Olender, 2009, p. 16). Au cours du XIX ${ }^{\mathrm{e}}$ siècle, cette acception se banalise. L'on cartographie alors les couleurs de peau de manière à produire l'«effet de "vérité" » nécessaire au maintien de l'ordre colonial (Vergès, 20ı3, p. 77).

Ces significations de la «race» seront mises à mal, au cours du $\mathrm{Xx}^{\mathrm{e}}$ siècle, par des avancées scientifiques, notamment dans le domaine de la génétique, qui n'affectent toutefois que partiellement les usages du terme. Comme le souligne le sociologue péruvien Anibal Quijano, «pour la majorité écrasante de la population mondiale, y compris les adversaires et les victimes du racisme, l'idée même de "race", comme un élément de la "nature" ayant des implications pour les rapports sociaux, se maintient, quasiment intacte, depuis ses origines» (2007, p. II2). En France, les catégories «indigène» puis «immigré» se substituent progressivement à la notion de race pour désagréger les mobilisations de classe. C'est dorénavant la «culture » ou «l'ethnicité » qui surdéterminent les individus (Balibar et Wallerstein, 1997) et légitiment les rapports structurels de domination.

Il faut donc traquer les expressions actuelles du racisme en s'intéressant aux modes de classement qui organisent et naturalisent les groupes humains (Fassin, 2006). Les écoles de pensée française et anglo-saxonne peuvent, à ce propos, être distinguées. Plusieurs chercheurs anglo-saxons soutiennent ainsi, à l'instar de Ruth Benedict (1942), que la race en tant que taxinomie fondée sur l'hérédité relève du champ scientifique alors que le racisme en tant que système doctrinaire s'oppose, quant à lui, toujours aux critères de scientificité, niant ainsi l'idée d'un lien entre attributs intellectuels et raciaux. La race est dorénavant considérée par les scientifiques anglo-saxons comme une construction sociale qui prend appui sur des attributs phénotypiques et détermine des rapports sociaux. En France, en revanche, des tabous entourent l'usage du terme: la catégorie «race» est une erreur, une faute de parcours, la trace d'une histoire peu valorisante mais aujourd'hui dépassée. Il n’en demeure pas moins que l'«universalisme» français paraît porteur de tensions favorables au développement du racisme : il est au cœur du travail d’unification de la réalité 
qui caractérise le nationalisme (Boltanski, 20I2). De tels soubassements idéologiques ont des implications concrètes. Pourtant, on ne peut que constater un écart quantitatif conséquent entre les travaux portant sur l'idéologie raciste et ceux centrés sur ses manifestations plus tangibles. L'on peut souligner, à ce propos, la faiblesse des approches empiriques du racisme (Eberhard, 2010).

\section{LE RACISME EN PAROLE ET EN ACTE}

Le racisme est un rapport social qui prend corps dans une multitude de situations : actions, jeux de relation et représentations sociales sont autant de dimensions à saisir pour établir une spectrographie du racisme. Si ces manifestations sont propres à des situations particulières d'oppression, elles n'en ont pas moins une origine commune. La caricature de Christiane Taubira parue en Une du journal Minute s'inscrit ainsi dans un socle de représentations en lien avec l'animalité des «Noirs». Elle fait, en cela, écho aux «cris de singe» qui ponctuent certains matchs de football (Bodinas, Robène et Héas, 2008). La parole des acteurs de la vie sociale représente un angle d’approche privilégié pour saisir la logique qui sous-tend ces expressions : elle apporte, d'une part, un éclairage sur la façon dont les individus interprètent les situations vécues tout en révélant, d’autre part, des pans de l'expérience des individus minorisés. Les discours participent, en effet, d'une atmosphère qui peut s'avérer oppressante. Bien que souvent saisis dans le cadre de la situation artificielle créée par les interactions entre enquêteur et enquêté, ils n'en révèlent pas moins la manière dont la question de l'altérité s'intègre dans un ensemble plus large auquel elle confère un sens spécifique. Que nous disent-ils des liens entre les avatars contemporains de l'idée de «race», le racisme et la racialisation dans la France contemporaine? Afin de l'envisager, nous étudierons, tout d’abord, les propos de deux femmes sexagénaires, Andrée et Catherine, pour caractériser l'emprise d'un référentiel racial en lien avec le «racisme de la chute et de l'exclusion sociale» (Wieviorka, 1993). Nous nous intéresserons, ensuite, aux propos de Pierre, 70 ans, et verrons comment le racisme peut également s'immiscer dans une configuration marquée par des rapports de domination. Nous questionnerons, enfin, à partir du discours d'individus plus jeunes, les 
particularités d'un rejet centré non plus sur l'idée de race, mais sur la culture, la religion musulmane et/ou la nationalité.

Andrée a 62 ans. Au moment où nous l'avons interrogée elle n’avait plus de logement et résidait dans un $\mathrm{CHRS}^{7}$ du sud de la France. Ses propos révèlent, outre une trajectoire de vie marquée par de nombreuses difficultés, la prégnance de représentations naturalisantes de l'Autre:

La vie elle est très dure avec l'euro. La première année, on l'a pas senti parce que les gens se libéraient de leurs francs, ils connaissaient pas encore trop bien l'euro donc ça marchait. Ça marchait même très bien. La seconde année ça commençait à baisser. La troisième année, c'était la catastrophe. Voyant ça, ils se sont quand même permis de laisser s'installer... en tout et pour tout il, y a quand même quinze Chinois! Quinze Chinois! Bon, déjà il y avait déjà les petits magasins des... des... des... Musulmans. [...]. Ça, c'est un truc... C'est un truc de merde. S'il y a un truc qui est libre, qu'ils en mettent un peu, mais enfin, quinze comme ça! Ils massacrent tout le monde! Et comme ils ont le fric... parce qu'entre eux c'est un peu comme dans le système des Juifs avant: ils sont solidaires les uns des autres, ils s'aident. [...] La France va devenir pauvre. On dégringole les marches. On va devenir au seuil de la pauvreté. On a trop voulu prendre, prendre... On était la vache à lait de tout le monde. Ils viennent en France d'ici, de là-bas... [...] Maintenant, c'est le piston. Pour les appartements c'est pareil: c'est le piston. [...] Quand on doit gouverner des gens de différentes ethnies, on ne doit pas favoriser plus les ethnies qui arrivent et laisser ceux qui sont là sur le pavé. Parce quavec leurs histoires de «touche pas à mon pote», de ceci, de cela, ils ont tous les avantages. [...] Est-ce que vous avez vu une Fatma dehors, vous? Jamais j'ai vu une Fatma dehors, ou faire la manche ou... Jamais. Par contre, des Français ou des... machins, oui. Déjà, nous, on n'a pas des tribus de gosses... [...] Faut pas se voiler. Moi, ce que je dis c'est pas du racisme [...]. Ce qui va arriver, c'est qu'ils vont nous bouffer. Ça, ça va arriver. Parce quaprès ça va devenir chez eux ici.

L'une des caractéristiques du discours raciste, outre les articulations entre social et naturel qu'il effectue, est qu'il est toujours surchargé d'un imaginaire qui traduit l'emprise des conceptions naturalisantes du social. Dans le discours d'Andrée, «Chinois » ou «Arabes » représentent des entités distinctes qui occupent une place spécifique dans un espace social par ailleurs marqué 
par des formes assez violentes de sélection. Les représentations de ces groupes sont empreintes du fantasme de la reproduction et de formes spécifiques de pouvoir. Les logiques de différenciation et les représentations du monde social s'informent ici mutuellement : la place que «Chinois » et «Arabes» occupent dans l'univers qu'elle décrit, révèlent des perceptions profondément pessimistes du monde social. La référence aux «ethnies» va ici de pair avec le déni de racisme. Les propos d’Andrée indiquent, ce faisant, la légitimité d’un mode de classement social - l'ethnicisation - qui permet de saisir et de donner sens aux inégalités (Vourc'h et de Rudder, 2006) : la référence aux catégories ethniques donne un visage au ressentiment induit par l'expérience de la précarité en désignant des boucs émissaires.

Le discours d'Andrée fait, en cela, écho aux analyses de Michel Wieviorka (1993) sur le racisme de la chute et de l'exclusion sociale. Les différenciations naturalisantes effectuées par Andrée - que révèlent l'emploi des catégories «Chinois » ou «Arabes»- ont des fondements sociaux qu'elles tendent toutefois à évacuer. Ses propos apparaissent, en cela, très proche du racisme classique. Car l'imaginaire qui transparaît dans le discours d'Andrée est celui de la race, soit d'une construction classificatoire élaborée à partir de traits héréditaires (Benedict, 1942). Le racisme classique s'appuie sur une conception des races comme entités biologiques distinctes et profondément inégales, physiquement comme intellectuellement. Cette conception a trouvé une caution «scientifique» dans les travaux de l'anthropologie physique (Badou, 2000). $\mathrm{Au} \mathrm{Xx}^{\mathrm{e}}$ siècle, les évolutions scientifiques ont rendu ces thèses caduques : le lien entre les capacités (intellectuelles, notamment) d'un individu et ses caractéristiques somatiques n'est désormais plus avalisé scientifiquement. La «race » n’en constitue pas moins, aujourd'hui encore, un référentiel implicite pour les individus exposés, au cours de leur enfance, aux théories racialistes et susceptibles de les réactiver pour donner sens à leurs difficultés.

Les propos de Catherine, 68 ans, rapatriée d'Algérie, qui nomme ses voisins clichois originaires du Maghreb, du Moyen-Orient ou d'Afrique subsaharienne, les «cafards» lorsqu'elle évoque avec irritation les nuisances dont ils seraient les auteurs, peuvent permettre d'envisager plus précisément les 
ressorts d'un tel racisme. «Avant, le quartier, ça allait. Mais depuis quelques années ils ont laissé s'installer les cafards. Ils jettent leurs poubelles par les fenêtres, ils sont sales, sales, sales... », souligne-t-elle ainsi à plusieurs reprises au cours de l'entretien. Ses difficultés quotidiennes ne semblent prendre sens qu’au regard d'un référentiel qui s'est constitué pour distinguer les populations mises en contact du fait des activités liées à l'esclavage et à la colonisation (Vergès, 20I3). Pour Catherine et pour d’autres résidents du Chêne-Pointu à Clichy-sous-Bois de même génération, les caractères somatiques constituent les principaux marqueurs différentiels mobilisés pour instituer une distance entre soi et l'image dévalorisante du quartier. Ici comme dans les sociétés étudiées par Jean-Luc Bonniol, les «traits physiques porteurs de contraste» permettent de naturaliser la «distance perçue» (2008, p. 23-25).

Le maintien de telles frontières revêt également un intérêt dans le cadre de relations plus directement marquées par la domination des individus racisés. Prenons appui, à titre d'exemple, sur les propos de Pierre, dentiste à la retraite âgé de 70 ans:

Le Maghrébin, le Maghrébin, moi, j’ai des copains bicots, il n’y a pas de problèmes. J'emploie le terme bicot, ce n'est pas péjoratif, hein. Je l'emploie comme je dirais un rital ou un ricain ou un flic en parlant de la police, ce n'est pas péjoratif [...]. Il y a des clodos, hein, qui, eux, viennent ici pour avoir des pensions, qui ne veulent pas travailler.

Si le vocabulaire mobilisé ne se distingue ici que peu de celui de Catherine ou d'Andrée, il n'exprime toutefois ni la colère ni la peur. Il annonce plutôt une tendance à l'infériorisation qui s'appuie sur des représentations relatives aux prédispositions à l'assistanat et à la délinquance des « Maghrébins ». Au cours de l'entretien, Pierre développe, en effet, l'idée que ces derniers auraient besoin d'être encadrés, «menés d'une certaine façon», pour ne pas céder à ces penchants naturels qui les conduiraient à l'assistanat ou à la délinquance. L'on peut voir là l'expression d'un racisme d'infériorisation qui va de pair, une fois de plus, avec un déni du racisme : il ne s'agit pas, en effet, pour Pierre d'exclure les individus ainsi désignés mais plutôt de légitimer qu’un traitement différencié leur soit administré en se référant à des caracté- 
ristiques propres au groupe discriminé (telles, ici, que l'assistanat). Il évoque ainsi longuement la nécessité de recourir à une main d'œuvre peu qualifiée pour faire fonctionner certains secteurs de l'économie. L’Autre est accepté en tant qu'il génère des profits mais un traitement préférentiel peut être accordé aux membres de l'endogroupe lorsque le besoin s'en fait sentir.

Cette vision s'inscrit dans la lignée d’une conception spécifique de l'universalisme, sous-tendue par l'idée que «le monde blanc serait porteur d'un projet de civilisation et de valeurs qui seraient universels » (Khiari, 20I3, p. 42). Étienne Balibar distingue, à ce propos, les fondements religieux, scientifiques et humanistes de l'universalisme (2005). Aux XVIII et XIX siècles, les théoriciens de la race se sont, en effet, inscrits en rupture avec l'idée biblique selon laquelle tous les hommes seraient « les fils d'un même Père » (Taguieff, 2008, p. 175). Ils ont soutenu, dans cette optique, une vision polygéniste. Autrement dit, la volonté de rompre avec l'universalisme religieux a conduit aux dérives de cet universalisme scientifique qui s'exprime dans les travaux de l'anthropologie physique et a pu s'inscrire dans une perspective évolutionniste : il s'agissait alors de mesurer l'écart séparant les autres races de la race blanche. Corollaire à l'industrialisation, le racisme d'infériorisation a pu mobiliser certaines de ces «figures de la pensée raciale» (voir Taguieff, 2008). Le racisme d'infériorisation est, selon Étienne Balibar et Immanuel Wallerstein (1977), lié au développement du capitalisme et à l'entreprise coloniale. Il représente un moyen d'abaisser le coût du travail de certains en contournant les normes méritocratiques tout en accordant «au groupe-victime une place dans la société à condition que ce soit la plus basse » (Wieviorka, 1993, p. 9). Ce racisme remplit une fonction à la fois sociale, en valorisant par opposition les individus de l'endogroupe, et économique, en réduisant les coûts de toute une partie de la force de travail. Il représente, à ce titre, un intérêt renouvelé à l'heure d'une remise en question des fictions égalitaristes et méritocratiques (Sennett, 2005; Martuccelli, 1995) accrue par l'expérience conjuguée du néolibéralisme et de la crise (Dardot et Laval, 2009). Sur les terrains investigués, il transpire dans les propos de plusieurs individus dont la situation peut apparaître, par certains aspects, privilégiée. 
Ce racisme d'infériorisation diffère en cela du racisme qui pointe parfois dans les propos de ces jeunes femmes précarisées d'une vingtaine d'années interrogées à Reims et dans le sud de la France et pour lesquelles une union avec un homme «d'origine différente», «Maghrébin» par exemple, n’est pas envisageable :

Je n’envisage pas, moi, de me marier avec... avec quelqu'un qui a une autre culture, différente de la mienne. Je ne sais pas pourquoi, bon, ça aurait beaucoup d'avantages de connaître une autre culture mais, non. Moi, je veux juste la mienne de culture, pas les autres!

Dans ces discours comme dans d'autres portant sur l'école, le poids de la dissemblance culturelle apparaît tel qu'il légitime, aux yeux de nos interlocuteurs, des pratiques différentielles :

Il faut se mettre aussi à la place des instructeurs. Quand - moi, je sais que j'en ai eu l'exemple précis - la maitresse si elle donne une punition ou elle fait faire quelque chose à un élève dont les parents ont les mêmes mœurs et les mêmes habitudes, même sans parler de religion, je parle juste d'état d'esprit. Un enfant qu'elle connaît, qui a les mêmes mœurs, elle pourra lui parler facilement, lui dire : «Là, tu as mal fait. Je te punis ». Alors que les autres, elle ne peut pas. Elle ne peut pas parce que... Parce que, déjà, elle ne peut pas trop leur donner de leçons, leur donner de conseils, et tout, parce que sinon tout de suite elle est en désaccord avec les parents. Vous comprenez? Donc, elle est obligée de les préserver. Mais les préserver ça veut dire moins leur faire la leçon.

Ces discours sur le mariage ou l'école révèlent de manière plus ou moins explicite la nécessité de maintenir à l'écart ou d'exclure l'Autre en raison de différences menaçantes. Ils évoquent, par plusieurs aspects, le racisme différentialiste défini par Pierre-André Taguieff (1988) pour mettre l'accent sur la facette la plus sombre des processus de différenciation. Parmi les cinq traits distinctifs de ce néoracisme mis en saillance par le philosophe, soulignons ici l'absence de «site politique fixe» (Taguieff, I986, p. IOI), qui facilite toutes les récupérations politiques, et l'omniprésence du thème de la différence. Pour certains de nos interlocuteurs, cette différence serait telle que le modèle républicain ne serait en mesure ni de la prendre en charge, ni de la gommer. Cette dernière conception va souvent de pair avec l'idée que les fondements culturels de la nation seraient menacés (Wieviorka, 1993). 
Dans ces expressions du racisme, l'association entre fait migratoire et décadence est récurrente mais rarement explicite. À travers elle, s'expriment parfois des enjeux liés à la définition des différences à l'heure de la globalisation. Les discours s'inscrivent alors dans un conflit particulier entre une vision organique du social et une conception libérale et guerrière parfois nommée «darwinisme social» (Taguieff, 1989). Porté par des mouvances nationalistes dont la base électorale peut fleurir à l'ombre de l'instrumentalisation de la condition minoritaire (Terrazzoni, 20I4) ${ }^{8}$, ce racisme trouve dans l'islamophobie une modalité d'expression renouvelée. Le déchaînement islamophobe qui a accompagné, au cours des années 2000, le «long débat autour du voile islamique » (Lorcerie, 2007, p. 330) trouve aujourd'hui de nouveaux espaces d'expression avec la médiatisation de l'engagement de jeunes français dans les conflits syriens ou irakiens. Ces expressions revêtent plusieurs traits typiques du racisme différentialiste: outre leur focalisation sur la différence et leur plasticité idéologique, elles se caractérisent par l'omniprésence des références aux «discours des autres», elle-même au cœur d'une stratégie discursive de «rétorsion», dont l'argument central produit « deux effets corrélatifs : délégitimation de l'adversaire, par le fait même de se légitimer au moyen de l'instrumentation discursive de celui-ci» (Taguieff, 1986, p. IOI). Ce racisme semble, en outre, s'exprimer d'autant plus facilement qu'il s'inscrit dans le cadre d'une critique convenue des religions. Dans un même temps, il déborde largement la question religieuse (Mestiri, Grosfoguel et Soum, 2008a) pour, in fine, «justifier des pratiques discriminatoires vis-à-vis des Musulmans et leur exclusion dans une société où ils constituent une minorité » (Martin-Muñoz, 2008, p. I6).

8 Cette tendance apparaît de manière particulièrement significative dans un communiqué du mouvement indépendantiste corse U Rinnovu (fondé en 1988): «Aujourd'hui, les Corses non reconnus et sans moyens politiques, culturels, économiques et sociaux pour intégrer les flux importants d'arrivants, deviennent minoritaires sur leur propre terre. [...] Du fait de la mondialisation libérale, du néo-colonialisme et du déséquilibre Nord-Sud, nous recevons une forte immigration essentiellement pour raison économique. [...] On se dirige donc vers un modèle d'intégration à la française, en échec depuis des années, avec sa recherche désespérée d'une citoyenneté républicaine française qui débouche de fait sur des communautés figées autour de leurs cultures» (Corse Matin, 2004, p. 2). 
Ces différentes expressions du racisme mobilisent et réinjectent dans l'espace des débats des conceptions sous-tendues par différentes logiques. Si ces discours ne constituent finalement que des mots, ils peuvent toutefois être envisagés à la fois comme des avatars des systèmes de représentations, et comme des facteurs influant sur l'expérience des Autres. Ils sont, en outre, susceptibles de s'articuler à des pratiques (violences, discrimination, ségrégation, etc.) qui limitent les capacités d'action des individus différenciés. Le racisme différentialiste, qui repose sur une vision belliciste des rapports sociaux, ouvre ainsi la voie aux violences islamophobes observées par le Collectif contre l'islamophobie en France (Dorémus et Mohammed, 20I3). Ces trois aspects que constituent discours, représentations et expériences ne peuvent d'ailleurs être déconnectés quau risque de faire le jeu d'une dénonciation parfois convenue d'un racisme qui resterait limité aux classes populaires, voire d'une confusion entre racisme et tensions interethniques qui tendrait à amoindrir l'expérience vécue du racisme du point de vue des auteurs comme des victimes (Noiriel, 2007b).

\section{VERS DES FORMES RENOUVELÉES DE RACIALISATION?}

Les discours racistes indiquent l'existence d'un imaginaire qui assure la jonction entre les registres de la nature et du social et qui invite à distinguer racisme et ethnicisation. C'est à travers cette jonction, qui est aussi fusion et production de nouveaux alliages, que s'opèrent les métamorphoses contemporaines de la haine de l'Autre, au-delà de la déconnection entre «race» et «racisme». Dans quelle mesure cette désarticulation contribue-t-elle au renouveau du racisme? Après avoir retracé les grandes étapes de cette disjonction et les clivages socio-économiques et politiques qui l'accompagnent, nous envisagerons quelles sont les particularités françaises en la matière.

La montée en puissance des catégories ethnico-raciales dans la sphère publique comme dans la sphère des interactions quotidiennes a été observée par plusieurs chercheurs au cours de la dernière décennie (Felouzis, 2003; Sajas, 20ı). Doit-on voir ici la traduction, dans le langage, des évolutions d'une société devenue «plurielle, aux racines culturelles multiples » (Gèze, 2006, p. 92) ou le reflet d’enjeux économiques et politiques? Les ruptures opérées par 
Nicolas Sarkozy lors de la campagne présidentielle de 2007 puis durant son quinquennat ont contribué, sous couvert dans un premier temps de «discrimination positive» (Simon, 2007, p. 153) puis de promotion d'un «républicanisme à la française » (Laborde, 20ı, p. 4), à banaliser des catégories ethnicoraciales et religieuses pour expliquer les problèmes sociaux. Outre leur caractère tabou, toujours transgressé, ces catégories se distinguent du registre de l'ethnicité par le caractère radicalement réifié des différences auxquelles elles se réfèrent plus ou moins explicitement : la «couleur » plutôt que la culture, ou encore la «culture » comme caractère héréditaire et transmissible.

Il faut donc casser le binôme race-racisme pour penser des frontières qui s'avèrent aujourd'hui en France encore prégnantes: en premier lieu, la croyance en des différences naturelles, biologiques, qui constitueraient des groupes, subsiste (Fassin, 2006, p. 30); en second lieu, des formes radicales de réification culturelle participent d'une surdétermination des traits, elle-même plus proche du registre du racisme que de l'ethnicité. Pourtant, la race ne peut être considérée comme un alibi parmi d’autres: elle est porteuse d'une puissance évocatrice spécifique et représente «l’instrument de domination sociale le plus efficace inventé ces 500 dernières années» (Quijano, 2007, p. II). Cette efficacité repose sur plusieurs de ses caractéristiques. Tout d’abord, les représentations que cristallise la notion sont toujours plus ou moins anachroniques. Non seulement l'idée de race s'oppose souvent à l'état des connaissances à un moment donné (Olender, 2009), mais elle s'appuie en outre sur des processus de corrélation entre visible et invisible qui perdurent dans le temps : des taxinomies basées sur les correspondances entre climat et tempérament qui prévalaient durant l'Antiquité (Pinna, 1989) à celles de l'anthropologie physique (Badou, 2000), des liens similaires entre physique et métaphysique sont établis. Aux éléments qui viennent contrarier la vision racialisante, il est toujours possible d'opposer le soupçon de caractères invisibles et/ ou masqués, en sorte que le racisme parvient à se maintenir, indépendamment d'une réelle croyance en l'existence de races distinctes. C'est ici que se cristallisent les angoisses concernant l'exercice réel du pouvoir et que prennent source les théories complotistes (Boltanski, 20I2) ou encore la tentation antisémite 
(Wieviorka, 2005). Et c'est ainsi que se développent aussi, à l'ombre de l'univers rationnel des discours scientifiques, de nouvelles formes de racialisation.

Le travail de terrain réalisé en Corse au cours des années 2000 a ainsi montré que les représentations relatives au «sexisme» des «Maghrébins» avaient une incidence forte sur les relations amoureuses de jeunes nés en Corse de parents français dont le seul marqueur différentiel semblait être le patronyme. Dans un tel contexte, être «arabe» constitue pour ces jeunes une identité assignée - parfois réappropriée - en raison d’une différence liée à un patrimoine héréditaire. Leurs expériences portent l'empreinte de cette racialisation,

Les discours publics qui ont accompagné les violences urbaines de 2005 invitent aussi à penser, bien au-delà du cas corse, la prévalence des présupposés raciaux dans la France contemporaine. Avec ces événements comme avec la médiatisation, plus récente, de l'engagement de jeunes «djihadistes» en Syrie ou en Irak (L’Express.fr, 20I4), c'est la violence des jeunes «arabes » et «noirs » qui est mise en scène et suscite l'effroi. Dans l'espace public français, «un réseau de relations causales »-l'absence d'éducation parentale, la violence viriliste, etc. - «font tenir les uns aux autres les événements auxquels l'expérience est confrontée » (Boltanski, 20I2, p. 22), contribuant à réactualiser et à renforcer le stigmate dont sont porteurs ces jeunes. Ils font alors l'expérience d'une «expulsion racialiste» (Mansouri,20I3, p. I34) renouvelée, sur la base de «caractéristiques phénotypiques spécifiques» (ibid., p. 135) mais aussi, et surtout, à partir des caractéristiques morales qui leur sont associées : « le "fatalisme”, le "puérilisme mental”, l’absence "d’appétit scientifique”, l'“immodération”, la "suggestibilité" et la soumission aux instincts » (ibid., p. I63). Ce sont ces caractéristiques qui, transmises de manière héréditaires, légitimeraient la nécessaire mise à l'écart ou le rejet.

Si la racialisation ne se traduit pas toujours par de telles pratiques, elle constitue toutefois une modalité de plus en plus répandue pour caractériser les problèmes sociaux (Noiriel, 2007a). À Clichy-sous-Bois, certains habitants installés depuis plus de vingt ans dans le quartier du Chêne Pointu assimilent ainsi la dégradation de leur copropriété à l'arrivée des «Noirs». La racialisation apparaît ici comme une modalité de gestion d'une ambivalence (Sanchez-Mazas, 2004, p.6) assez proche du racisme: elle se nourrit des tensions entre idéal égalitaire et dévelop- 
pement des inégalités et sappuie, en outre, sur des catégories voisines découlant d'un même processus de hiérarchisation.

Ce faisant, la racialisation est susceptible d'éclairer les ressorts de ce nationalisme défensif qui a entretenu, au cours des derniers siècles, des liens étroits avec le racisme (Wieviorka, I993; Noiriel, 2013). Les propos de Farida, 22 ans et résidant dans le quartier de l'Alma à Roubaix peuvent permettre d'illustrer ce dernier point :

Il y en a qui viennent du bled, ils trouvent plus de travail que nous. Ils vivent mieux que nous, je ne trouve pas ça normal, ils ont plus d'aides que nous [...] [Vous avez l'impression que ce n'est pas juste?] Oui, voila: c'est pas juste. En fait, ils savent à qui donner [...] Depuis que je suis née je vais à la mission locale, j’ai toujours été pointée du doigt. Pourquoi cette aide qui est sortie, moi on me la refuse ? [...] C'est pour ça qu'il y a du bordel, que les gens crament les voitures. [... ] Y a rien [...] Il y a rien, Madame. Vraiment. [Rien du tout ?] Peut être un peu pour les garçons et encore pas beaucoup: peinture, maçonnerie mais sinon, après, il y a pas.

Dans ce discours, la principale ligne de fracture semble être celle qui distingue le «Nous Français», stigmatisés et délaissés du «Eux Immigrés», aidés. C'est elle qui donne sens au sentiment d'injustice et permet à Farida d'identifier un coupable : l'immigré. Ce dernier discours permet d'illustrer les effets de renforcement possible entre racialisation et racisme dans un contexte fortement concurrentiel et marqué par l'expérience des inégalités. Ces propos s'inscrivent dans le sillon des discours, originellement d'extrême-droite qui se sont banalisés en France au cours des années 1980 (Bonnafous, I99I) et transparaissent souvent là où le nationalisme sourd (Taguieff, 1986) en s'appuyant sur un sentiment de trahison catégoriel. Dans cette dernière configuration l'on perçoit le caractère profondément modulaire d'un racisme susceptible de s'attaquer à tous les «groupes qui sont sociologiquement en situation de dépendance ou d'infériorité » (Guillaumin, 2002 [1972], p. 94, souligné par l’auteur). 
La prégnance des enjeux médiatique et politique qui sous-tendent les usages du concept de «racisme» dans l'espace public comme les évolutions scientifiques et sociétales qui ont contribué sinon à la disparition, du moins à la dé-légitimation de la «race», conduisent à questionner la valeur opératoire du concept de racisme. Doit-on, pour caractériser les nouvelles manifestations du rejet dont le pendant politique est l'émergence d'une droite «décomplexée » (Ivaldi, 2007, p. I20), parler de «racisme différentialiste» ou voir plutôt ici les effets de la «racialisation»?

Une controverse concernant le caractère - homogène ou hétérogène - du racisme a marqué l'histoire de la pensée du racisme au cours des années 1980. Elle a atteint son paroxysme lors du $3^{\mathrm{e}}$ colloque international de lexicologie politique (Taguieff, 2008) et opposé des chercheurs soulignant le caractère profondément «syncrétique» du racisme en acte (Guillaumin, 2002 [1972], p. 40) aux tenants d'un néo-racisme, différentialiste (Taguieff, 2006). Si l'on accepte de souscrire à l'idée d'une distinction des différentes logiques racistes à visée analytique (Wieviorka, I999 [1998]), l'on peut alors questionner les particularités d'un racisme différentialiste qui a permis de renouveler la fonction première du racisme classique - légitimer l'exclusion et la mise à distance - en substituant la «culture» à la «race». Il offre ainsi une grille de lecture qui s'inscrit dans la continuité du darwinisme social. Sur les terrains investigués, cette logique coexiste avec des logiques d'infériorisation qui permettent, quant à elles, de légitimer le contournement des normes égalitaristes et méritocratiques. Ces deux logiques cohabitent en dépit des avancées scientifiques qui ont remis en question l'idée de différences héréditaires physiologiques et morales. Ce dernier point permet de souligner le caractère hybride et modulaire du racisme. L'essentialisation des différences sociales permet, ainsi, de masquer à la fois les tensions consécutives au développement du capitalisme et celles, plus spécifiquement françaises, liées aux contradictions inhérentes à l’«universalisme», au «nationalisme» et aux liens que ces deux termes entretiennent. $\mathrm{Si}$, sur ce dernier point, la vigilance concernant les biais induits par un «nationalisme méthodologique» qui consiste bien souvent à «comprendre le monde social en prenant l'État-nation pour unité d’analyse » (Dumitru, 20I4, p. 9) doit 
prévaloir, la prise en considération de différents niveaux de contextualisation peut permettre d'échapper à ces écueils (Poiret, 201 I).

L'on peut ainsi distinguer une racialisation des discours publics largement instrumentalisées par les acteurs politiques ou économiques d'une autre, observée, quant à elle, dans les quartiers populaires, qui témoigne de mécanismes de dédramatisation ou de resignification des acteurs sociaux, qui prennent euxmêmes sens dans l'expérience du racisme (Poli, 2005). Çà et là, la réification des différences ouvre toutefois de nouveaux espaces à un racisme profondément autoréférentiel. Là encore, il faut souligner le rôle très spécifique joué par le «nationalisme à la française » et, plus spécifiquement, par la manière dont ce nationalisme recouvre parfois les différences sociales tout en favorisant ce sentiment de trahison catégoriel qui ouvre la voie au racisme de la chute et de l'exclusion. Bien que pris dans des enjeux politiques et médiatiques, le concept de «racisme» permet donc aujourd'hui encore d'embrasser dans un même effort de compréhension des manifestations (discours, violences, etc.) et des logiques plurielles. Les réflexions produites à ce propos invitent de surcroît à considérer comment il conjugue une fonction triple: donner sens aux inégalités, légitimer les rapports structurels de domination et agréger des collectifs.

\section{BIBLIOGRAPHIE}

AUSTIN John L., 199I, Quand dire, c'est faire, Paris, Éditions du Seuil, «Points essais».

BADOU Gérard, 2002, L'Énigme de la Vénus hottentote, Paris, Éditions Payot et Rivages. BALIBAR Étienne, 2005, «La construction du racisme », Actuel Marx, 2-38, p. II-28. - et WALLERSTEIN Immanuel, 1997, Race, Nation, Classe: les identités ambiguës, Paris, La Découverte.

BANCEL Nicolas et BLANCHARD Pascal, 1998, De l'indigène à l'immigré, Paris, Gallimard, p. 345.

BENEDICT Ruth, 1942, Race and racism, Londres, Routledge and Kegan Paul. BERTHELEU Hélène, 2007, «Sens et usages de "l'ethnicisation"», Revue européenne des migrations internationales, 23-2, p.7-28. 
BLANCHARD Pascal et BOËTSCH Gilles, 2013, «La Construction du "Blanc" dans l'iconographie coloniale», in LAURENT et LECLÈRE, 2013a, p. 128-139.

BODINAS Dominique, ROBÈNE Luc et HÉAS Stéphane, 2008, «Racisme, xénophobie et idéologies politiques dans les stades de football », Raisons politiques, 29-1, p. 147-167.

BOLTANSKI Luc, 2012, Énigmes et Complots: une enquête à propos d'enquêtes, Paris, Gallimard.

BONNAFOUS Simone, 1991, L'Immigration prise aux mots. Les immigrés dans la presse au tournant des années 1980, Paris, Kimé.

BONNIOL Jean-Luc, 2006, «Penser et gérer l'hérédité des caractères discriminants dans les sociétés esclavagistes et post-esclavagistes», Rives nord-méditerranéennes, 24, [en ligne, consulté le 21 septembre 2014]: <https://rives.revues.org/552>.

BUTLER Judith, 2004, Le Pouvoir des mots. Politique du performatif, Paris, Éditions Amsterdam.

-, 2006, Trouble dans le genre. Le féminisme et la subversion de l'identité, Paris, La Découverte.

CNCDH, 2013, «Rapport racisme, antisémitisme et xénophobie 2012: inquiétante montée de l'intolérance», Communiqué de presse, jeudi 21 mars.

Corse Matin, 2004, «Maîtrise de la politique migratoire. Libre opinion de Rinnovu», mercredi 10 novembre, p. 2.

DARDOT Pierre et LAVAL Christian, La Nouvelle Raison du monde. Essai sur la société néolibérale, Paris, La Découverte.

DEGIOANNI Anna et GOURJON Géraud, «La "Race blanche". Retour sur les tentatives trompeuses de classification et de hiérarchisation de l'espère humaine » in LAURENT et LECLÈRE, 2013a, p. 206-213.

DELPHY Christine, 2008, Classer, dominer. Qui sont les Autres?, Paris, La Fabrique.

DE RUDDER Véronique, 1991, «La recherche sur la coexistence pluriethnique. Bilan, critiques et propositions», Espaces et Sociétés, 64, p. I31-157. 
DORÉMUS Fabien et MOHAMMED Marwan, 2013, «Les Femmes sont les principales victimes de la violence islamophobe», Le Télescope d'Amiens, 19 décembre [en ligne, consulté le 13 janvier 2014]:

<http://blogs.mediapart.fr/edition/le-telescope-damiens/article/191213/ les-femmes-sont-les-principales-victimes-de-la-violence-islamophobe>.

DUMITRU Speranta, 2014, «Qu'est-ce que le nationalisme méthodologique? Essai de typologie», Raisons politiques, 54-2, p. 9-22.

EBERHARD Mireille, 2010, «De l'expérience du racisme à sa reconnaissance comme discrimination. Stratégies discursives et conflits d'interprétation», Sociologie, 4-I, p.479-495.

FASSIN Didier, 2006, «Nommer, interpréter. Le sens commun de la question raciale», in De la question sociale à la question raciale?, Didier Fassin et Éric Fassin (dir.), Paris, La Découverte.

GÈZE François, 2006, «Les "Intégristes de la République" et les émeutes de novembre ou les effets de la mutation médiatique de la figure de l'intellectuel», Mouvements, 4, mars avril, p.88-100.

GUILLAUMIN Collette, 2002 (1972), L'ldéologie raciste, Paris, Gallimard.

IVALDI Gilles, 2007, «L'Extrême-droite à la dérive: rééquilibrage du système partisan et recomposition à droite », Revue politique et parlementaire, 1044, juillet-septembre, p. 113-122.

JENKINS Richard, 2000, «Categorization: Identity, Social Process and Epistemology», Current Sociology, 48-3, p. 7-25.

KHIARI Sadri, 2013, «Nous ne voulons plus être les tirailleurs sénégalais d'aucune cause », in LAURENT et LECLÈRE, 2013a, p. 39-48.

L'Express.fr, 2014, «Syrie et Irak: le nombre de djihadistes européens en hausse», 23 octobre, [en ligne]: <http://www.lexpress.fr/actualite/monde/proche-moyenorient/syrie-et-irak-le-nombre-de-djihadistes-europeens-en-hausse_|578590.html>.

LABORDE Cécile, 2010, «Républicanisme critique vs républicanisme conservateur: repenser les "accommodements raisonnables" », Critique internationale, 3-44, p. 19-33. 
LAURENT Sylvie et LECLÈRE Thierry (dir.), 2013a, De quelle couleur sont les Blancs.

Des «petits Blancs» des colonies au «racisme anti-Blancs》, Paris, La Découverte.

-, 2013b, «Introduction 》, in LAURENT et LECLÈRE, 2013a, p. 7-22.

LUCAS Jean-Claude, ROSA Jérôme et LECLEC'H Janine, 20I2, CLR 1000 exercices de calcul mental CE2/CM - livre du maître, Paris, Hachette éducation.

MANSOURI Malika, 2013, Révoltes post-coloniales au cœur de l'Hexagone. Voix d'adolescents, Paris, PUF.

MARTIN-MUÑOZ Gema, 2008, «L'islamophobie inconsciente», in MESTIRI, GROSFOGUEL et SOUM, 2008a, p. 15-28.

MARTUCCELLI Danilo, 1995, Décalages, Paris, PUF.

MASCLET Olivier, 2012, Sociologie de la diversité et des discriminations, Paris, Armand Colin.

MESTIRI Mohamed, GROSFOGUEL Ramon et SOUM El Yamine, 2008a, Islamophobie dans le monde moderne, Saint-Ouen, IIIT France, p. 15-28.

-, 2008b, «Introduction», in MESTIRI, GROSFOGUEL et SOUM, 2008a, p.9-II.

NOIRIEL Gérard, 2007a, Immigration, antisémitisme et racisme en France (XIXe-XXe siècle). Discours publics, humiliations privées, Paris, Fayard, 2007, p. 130.

-, 2007b, Racisme: la responsabilité des élites, Paris, Éditions Textuel.

-, 2013, «Il n'y a pas de "question blanche" ... », in LAURENT et LECLÈRE, 2013a, p.34-38.

OLENDER Maurice, 2005, Race sans histoire, Paris, Galaade Éditions.

OMI Michael et WINANT Howard, 1993, «On the Theoretical Status of the Concept of Race», in Race, identity and representation in education, Cameron McCarthy and Warren Crichlow (dir.), New York, Routledge, p. 3-I0.

PINNA Mario, 1989, «Un aperçu historique de "la théorie des climats"», Annales de Géographie, 98-547, p. 322-325.

POIRET Christian, 20II, «Les processus d'ethnicisation et de raci(ali)sation dans la France contemporaine: Africains, Ultramarins et "Noirs"», Revue européenne des migrations internationales, 27-I, p. 107-127. 
POLI Alexandra, 2005, «L'expérience française du racisme et des discriminations raciales. De la condamnation morale à la prise en charge de la subjectivité des victimes», Thèse de sociologie, Paris, EHESS.

POUTIGNAT Philippe et STREIFF-FERRART Jocelyne, 1995, Théories de l'ethnicité, Paris, PUF.

QUIJANO Anibal, 2007, «"Race" et colonialité du pouvoir», Mouvements, 3-5I, p. $|1|-|| 8$.

RÄTHZEL Nora, 1998, «Rapport entre nationalisme, minorité ethnique, égalité et autodétermination. L'exemple de l'Allemagne » in Immigration et racisme en Europe, Andrea Réa (dir.), Paris, Éditions Complexe, p. 45-74.

SAJAS Cécile, 2010, «Éduquer contre le racisme et les discriminations au collège: tout un programme», Mémoire Master 2 recherche, Université Paris VII - Diderot.

SANCHEZ-MAZAS Margarita, 2004, Racisme et xénophobie, Paris, PUF.

SENNETT Richard, 2005, Respect. De la dignité de l'homme dans un monde d'inégalité, Paris, Hachette Littératures.

SIMON Patrick, 2007, «Comment la lutte contre les discriminations est passée à droite», Mouvements, 4-52, p. 153-163.

SPITZER Léo, 1948, «Ratio/race», in Id., Essays in Historical Semantics, New York, S. F. Vanni, p. 147-176

TAGUIEFF Pierre-André, 1986, «L'identité nationale saisie par les logiques de racisation. Aspects, figures et problèmes du racisme différentialiste $\gg$, Mots, 12 , p. $91-128$.

-, 1988, La Force du préjugé. Essai sur le racisme et ses doubles, Paris, La Découverte. -, 1989, «La Métaphysique de Jean-Marie Le Pen», in Le Front national à découvert, Nonna Mayer et Pascal Perrineau (dir.), Paris, Presses de la fondation nationale des sciences politiques, p. 173-194.

TERRAZZONI Liza, 2014, «La condition minoritaire corse ou la manipulation politique du paradigme de la minorité », DIRE, 5, [en ligne]: <http://epublications.unilim.fr/revues/dire/402>. 
VERGÈS Françoise, 2013, «La "Ligne de couleur". Esclavage et racisme colonial et postcolonial», in LAURENT et LECLÈRE, 20।3a, p.76-86.

VOURC'H François et DE RUDDER Véronique, 2006, «De haut en bas de la hiérarchie syndicale: dits et non-dits sur le racisme», Travailler, 2-16, p. 37-56.

WIEVIORKA Michel (dir.), 1992, La France raciste, Paris, Éditions du Seuil.

-, 1993, «Nationalisme et racisme», Cahiers de recherche sociologique, 20, p. I59-|8I.

-, 1999 (1998), Le Racisme. Une introduction, Paris, La Découverte.

-, 2005, La Tentation antisémite. Haine des Juifs dans la France d'aujourd'hui, Paris, Robert Laffont. 